Marquette University

e-Publications@Marquette

Mechanical Engineering Faculty Research and

Publications

Mechanical Engineering, Department of

2019

\title{
A Hybrid Droplet Vaporization-Chemical Surrogate Approach for Emulating Vaporization, Physical Properties, and Chemical Combustion Behavior of Multicomponent Fuels
}

\author{
Alanna Y. Cooney \\ Marquette University \\ Simcha L. Singer \\ Marquette University, simcha.singer@marquette.edu
}

Follow this and additional works at: https://epublications.marquette.edu/mechengin_fac

Part of the Mechanical Engineering Commons

\section{Recommended Citation}

Cooney, Alanna Y. and Singer, Simcha L., "A Hybrid Droplet Vaporization-Chemical Surrogate Approach for Emulating Vaporization, Physical Properties, and Chemical Combustion Behavior of Multicomponent Fuels" (2019). Mechanical Engineering Faculty Research and Publications. 239.

https://epublications.marquette.edu/mechengin_fac/239 
Marquette University

e-Publications@Marquette

\title{
Mechanical Engineering Faculty Research and Publications/College of Engineering
}

This paper is NOT THE PUBLISHED VERSION; but the author's final, peer-reviewed manuscript. The published version may be accessed by following the link in th citation below.

Proceedings of the Combustion Institute, Vol. 37, No. 3 (2019): 3229-3236. DOI. This article is (C) Elsevier and permission has been granted for this version to appear in e-Publications@Marquette. Elsevier does not grant permission for this article to be further copied/distributed or hosted elsewhere without the express permission from Elsevier.

\section{A Hybrid Droplet Vaporization-Chemical Surrogate Approach for Emulating Vaporization, Physical Properties, and Chemical Combustion Behavior of Multicomponent Fuels}

\author{
Alanna Y. Cooney \\ Department of Mechanical Engineering, Marquette University, 1637 W. Wisconsin Ave, Milwaukee, WI \\ Simcha L. Singer \\ Department of Mechanical Engineering, Marquette University, 1637 W. Wisconsin Ave, Milwaukee, WI

\section{Abstract} \\ The complex nature of multicomponent aviation fuels presents a daunting task for accurately simulating \\ combustion behavior without incurring impractical computational costs. To reduce computation time, chemical \\ fuel surrogates comprised of only a few species are used to emulate the combustion of complex pre-vaporized
}


fuels. These surrogates are often unable to match the vaporization behavior and physical properties of the real fuel and fail to capture the effect of preferential vaporization on combustion behavior. Therefore, a computationally efficient, hybrid droplet vaporization-chemical surrogate approach has been developed which emulates both the physical and chemical properties of a multicomponent kerosene fuel. The droplet vaporization/physical portion of the hybrid uses the Coupled Algebraic-Direct Quadrature Method of Moments with delumping to accurately solve for the evolution of every discrete species in a vaporizing fuel droplet with the computational efficiency of a continuous thermodynamic model. The chemical surrogate portion of the hybrid is linked to the vaporization model using a functional group matching method, which creates an instantaneous surrogate composition to match the distribution of chemical functional groups $\left(\mathrm{CH}_{2}\right.$, $\left(\mathrm{CH}_{2}\right)_{n}, \mathrm{CH}_{3}$ and Benzyl-type) in the vaporization flux of the full fuel. The result is a hybrid method which can accurately and efficiently predict time-dependent, distillation-resolved combustion property targets of the vaporizing fuel and can be used to investigate the effects of preferential vaporization on combustion behavior.

\section{Keywords}

Hybrid surrogate, Chemical functional group, Multicomponent droplet vaporization, Coupled Algebraic-Direct Quadrature Method of Moments, Delumping

\section{Introduction}

Computational efficiency is an important consideration when modeling spray combustion processes using computational fluid dynamics (CFD). Since hydrocarbon fuels are composed of hundreds of chemical species [1], [2], [3], [4], [5], [6], both the droplet vaporization and chemical kinetic models must be optimized for maximum accuracy with minimal computational expense [7]. A variety of surrogate development techniques [8], [9], [10], [11], [12], [13], [14], [15], [16], [17], [18], [19], [20] have been formulated which attempt to emulate the combustion behavior of a real fuel using only a small number of species. However, the complex nature of hydrocarbon fuels makes it difficult to successfully mimic both the physical properties and the chemical combustion kinetics of a real fuel using a single surrogate [7], [21], [22], [23], [24].

Existing methods to develop chemical surrogates for various pre-vaporized aviation fuels use optimization methods to calculate the surrogate composition in order to match combustion behaviors to those of a real fuel [8], [11], [15], [18], [19], [20]. Most recently, Won et al. extended previous combustion property target (CPT) matching methods to include four key CPTs: molecular weight (MW), hydrogen to carbon ratio $(\mathrm{H} / \mathrm{C})$, threshold sooting index (TSI) and derived cetane number (DCN) [19]. These CPTs have been demonstrated to be indicators of global combustion behavior [19]. By formulating surrogate compositions based on constrained CPTs, it was demonstrated that a strong correlation exists between the CPTs of a fuel and the distribution of chemical functional groups within the fuel [19]. Although these chemical surrogate methods have proven to be accurate and computationally attractive for pre-vaporized fuels, there remains a need for accurate modeling of fuel physical properties and further investigation into the effects of preferential vaporization on combustion behavior [21], [22], [23], [24]. The extent to which preferential vaporization can impact the evolution of key combustion properties of a fuel is an important unsolved problem [7], [23].

In order to account for the inability of chemical fuel surrogates to accurately model physical properties and vaporization behavior, a hybrid method was developed which pairs a physical surrogate to a separate chemical surrogate, to model vaporization and chemical kinetics, respectively [9], [10], [13], [16]. In order to link the physical and chemical surrogates, Krishnasamy et al. used a group chemistry representation method which grouped the physical surrogate species of each hydrocarbon class into a single representative chemical surrogate species [13]. The hybrid modeling concept improves the ability of surrogate methods to accurately 
emulate both the physical and chemical properties of a fuel. The challenge of these methods is accurately transitioning from the physical surrogate species to the chemical surrogate species within a CFD simulation.

The novel surrogate method developed in this paper takes a hybrid approach with separate vaporization and chemical surrogate models. In lieu of creating a relatively large physical surrogate based on experimental distillation curves, as was done in [13], the droplet vaporization process is modeled using the previously developed Coupled Algebraic - Direct Quadrature Method of Moments (CA-DQMoM) with delumping approach [25] for a droplet of known chemical composition. CA-DQMoM uses a computationally efficient continuous thermodynamics method (CTM) to accurately model multicomponent droplet vaporization with finite rates of liquid diffusion [25], and the subsequent delumping step [26] reconstructs information on each discrete fuel species at negligible computational cost. The new hybrid method links the physical vaporization model to the chemical surrogate composition by matching the distribution of key combustion functional groups identified by Won et al. [19]. The linking uses algebraic equations to compute the composition of the gaseous chemical surrogate at every time step from the results of the CA-DQMoM with delumping vaporization model. The overall approach is summarized in Fig. 1.

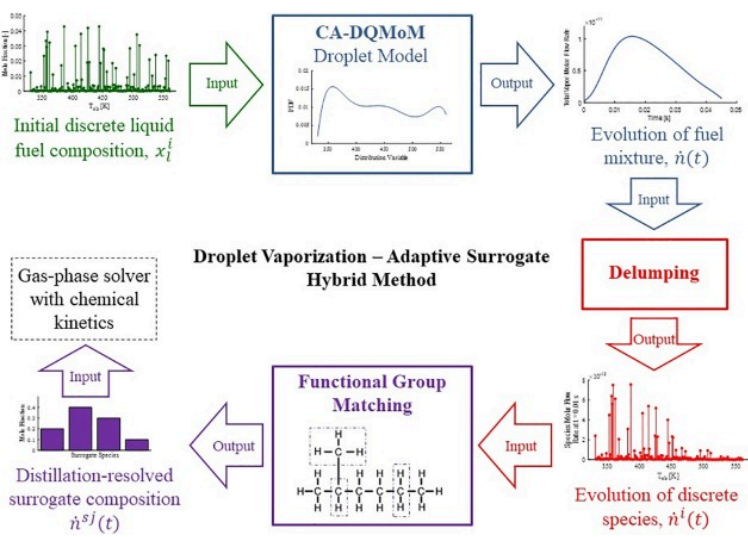

Fig. 1. Summary of the hybrid droplet vaporization - adaptive surrogate method using CA-DQMoM with delumping and FGM.

The first novelty of the method presented here is the use of CA-DQMoM with delumping as the physical portion of the hybrid model. Unlike a physical surrogate [10], [13], the computational efficiency of CA-DQMoM is not constrained by the number of discrete fuel components required to accurately model the physical properties of the fuel [25]. A second novelty of the paper is the development of the functional group matching (FGM) approach for linking the physical droplet vaporization model to the chemical surrogate to emulate the timedependent combustion behavior of the vaporizing fuel. The method is advantageous because instead of matching hydrocarbon classes, it matches the distribution of chemical functional groups which have been proven to be important indicators of combustion behavior [19]. The resulting computationally efficient hybrid method accurately models the time-dependent CPTs for a vaporizing multicomponent fuel droplet.

\section{Physical model for droplet vaporization}

\subsection{Discrete component model (DCM)}

The discrete component model (DCM) previously developed in [25] on a molar basis is the "exact" method used to validate the multicomponent droplet vaporization model for a kerosene fuel with known chemical composition. Differential and algebraic equations are solved for every discrete species within the droplet, making it accurate but computationally expensive [25]. The DCM accounts for finite rates of heat and mass transfer within the liquid droplet using quasi-steady temperature [2], [27] and species mole 
fraction [28] profiles. Assuming the mixtures are ideal, vapor-liquid equilibrium at the droplet surface is established by Raoult's law, as is commonly done in droplet vaporization models [2], [28], [29], [30], [31], [32], [33], [34]. The validity of the ideal mixture assumption was recently demonstrated for aviation fuels at low pressure [6]. The thermo-physical properties of each discrete hydrocarbon species are obtained from the appendix of [1] and DCM blending rules [2] are used to calculate the mixture properties. The model equations are the same as those previously defined in [25] and are therefore not provided in this paper.

\subsection{CA-DQMoM with node approximation and delumping}

The CA-DQMoM with delumping method developed in [25] is applied to the quasi-steady DCM framework to compute the evolution of every discrete species within the vaporizing multi-component droplet at a fraction of the computational time of the DCM. Quadrature moment methods [2], [26], [29], [30], [31], [35], [36] are a type of CTM which characterize the multicomponent mixture as a continuous function of a distribution variable, in this case normal boiling temperature, without assuming any functional form. DQMoM solves directly for the nodes (boiling temperatures) and weights (mole fractions) of an evolving equivalent distribution [36]. CADQMoM is unique in that it solves for two liquid mole fraction distributions to account for liquid diffusion within the droplet using a homogeneous DQMoM [36] approach coupled to algebraic moment equations [25]. The node approximation method [25] further improves computational efficiency by using the same set of nodes to characterize both distributions, for conditions where the far-field gas composition is either air or a percentage of stoichiometric fuel. A traditional limitation of CTMs is that they are unable to provide information on discrete species and only compute the evolution of the mixture as a whole. However, the subsequent delumping method, developed in [26], allows for the calculation of the evolution of each discrete species using numerical integration by linearizing the governing equation using the results of CA-DQMoM [25]. Both CA-DQMoM and delumping have been previously demonstrated to be accurate and computationally efficient for modeling multicomponent droplet vaporization [25].

The thermo-physical properties for the CA-DQMoM model are calculated from correlations for iso-alkanes in the appendix of [1] and CTM blending rules [2] are used to calculate the mixture properties. Although it is possible to employ separate distributions and property correlations for each chemical family, Laurent has shown that when normal boiling temperature is chosen as the distribution variable, the use of iso-alkane correlations for all components of kerosene is acceptable [2]. Since the DCM is calculated from discrete species properties for several families, the accuracy of this assumption is evaluated by the results of this paper. Because the CADQMoM and delumping model assumptions and equations are the same as those previously defined in [25], they are not provided in this paper.

\subsection{Droplet vaporization model output}

The output of both the DCM and CA-DQMoM with delumping models is the instantaneous vapor molar flow rate of every discrete species, $\dot{n}^{i}$, from the droplet. Without a subsequent surrogate method, the species vapor molar flow rates would serve as the CFD source terms for the $n$ discrete species entering the gas-phase.

\section{Chemical surrogate}

The novel chemical surrogate functional group matching (FGM) method is a simple algebraic system of equations which calculates the chemical surrogate source terms for the gas phase solver in a CFD simulation based on the output of the delumped CA-DQMoM droplet vaporization model developed in Section 2. The determination of the surrogate composition does not require optimization calculations, as has been done in formulating previous chemical surrogates, making it a computationally efficient method for solving a vaporization-dependent surrogate composition on the fly. Because the input to the $F G M, n \ddot{i}$, includes every 
discrete species of the fuel, it enables very accurate emulation of a complex multicomponent fuel in the gas phase by the chemical surrogate. The molar flow rates of the FGM chemical surrogate species are calculated by matching the distribution of functional groups to those of the full delumped fuel. The chemical functional groups correlated with combustion behavior were given by Won et al. as $\mathrm{CH}_{2},\left(\mathrm{CH}_{2}\right)_{n}, \mathrm{CH}_{3}, \mathrm{CH}, \mathrm{C}$, and Benzyl-type [19].

\subsection{Selection of chemical surrogate species}

The chemical surrogate components were selected based on species which are commonly used [13], [19] to ensure that chemical kinetic mechanisms are available for each surrogate species. Four components were selected: a lightweight n-alkane (n-heptane), a heavy n-alkane (n-hexadecane), an iso-alkane (iso-dodecane), and an aromatic (135 TMB). The two n-alkanes are needed to match the $\mathrm{MW}$ of the fuel, which will be discussed in the next section. The surrogate components and their functional group compositions are listed in Table 1.

Table 1. Chemical surrogate components and their functional groups.

\begin{tabular}{|l|l|l|l|l|l|l|l|l|l|}
\hline Class & Species & Index, $\mathrm{Sj}$ & Formula & $\mathrm{CH}_{2}$ & $\left(\mathrm{CH}_{2}\right)_{\mathrm{n}}$ & $\mathrm{CH}_{3}$ & $\mathrm{CH}$ & $\mathrm{C}$ & Benzyl \\
\hline n-alkane & n-heptane & $\mathrm{S} 1$ & $\mathrm{C}_{7} \mathrm{H}_{16}$ & 0 & 5 & 2 & 0 & 0 & 0 \\
\hline n-alkane & n-hexadecane & $\mathrm{S} 2$ & $\mathrm{C}_{16} \mathrm{H}_{34}$ & 0 & 14 & 2 & 0 & 0 & 0 \\
\hline iso-alkane & iso-dodecane & $\mathrm{S} 3$ & $\mathrm{C}_{12} \mathrm{H}_{26}$ & 2 & 0 & 7 & 1 & 2 & 0 \\
\hline aromatic & $1,3,5$-trimethylbenzene & $\mathrm{S} 4$ & $\mathrm{C}_{9} \mathrm{H}_{12}$ & 0 & 0 & 3 & 0 & 0 & 1 \\
\hline
\end{tabular}

\subsection{Functional group matching (FGM) method}

From the output of the droplet vaporization model, the discrete species vapor molar flow rate, $\dot{n}^{i}$, is used to calculate the molar flow rate of each functional group entering the gas phase:

(1) $\dot{n}_{k}^{F}=\sum_{i=1}^{n} \dot{n}^{i} G_{k}^{i}$

where $k$ is the index of the functional group, the superscript $F$ denotes for the total fuel, and $G$ is the number of moles of the functional group per mole of the species. For example, $G_{k=\mathrm{CH}_{3}}^{i}$ represents the moles of functional group $\mathrm{CH}_{3}$ per mole of discrete component $i$ and $\dot{n}_{k=\mathrm{CH}_{3}}^{F}$ represents the total molar flow rate of functional group $\mathrm{CH}_{3}$ calculated from the full delumped fuel. The functional groups $\mathrm{CH}_{2},\left(\mathrm{CH}_{2}\right)_{n}, \mathrm{CH}_{3}$ and Benzyl-type were selected for matching based on a previous sensitivity analysis [19]. In addition to the four key functional groups, the $\mathrm{MW}$ is used as a matching parameter. The molar flow rates of each chemical surrogate component, $\dot{n}^{S j}$, are calculated based on the following matching matrix:

$$
\left(2\left[\begin{array}{cccc}
0 & 0 & 0 & G_{\mathrm{Benz}}^{S 4} \\
\left(G_{\mathrm{CH}_{2}}^{S 1}+G_{\left(\mathrm{CH}_{2}\right)_{n}}^{S 1}\right) & \left(G_{\mathrm{CH}_{2}}^{S 2}+G_{\left(\mathrm{CH}_{2}\right)_{n}}^{S 2}\right) & \left(G_{\mathrm{CH}_{2}}^{S 3}+G_{\left(\mathrm{CH}_{2}\right)_{n}}^{S 3}\right. & 0 \\
G_{\mathrm{CH}_{3}}^{S 1} & G_{\mathrm{CH}_{3}}^{S 2} & G_{\mathrm{CH}_{3}}^{S 3} & G_{\mathrm{CH}_{3}}^{S 4} \\
\left(M W_{1}-M W_{F}\right) & \left(M W_{2}-M W_{F}\right) & \left(M W_{3}-M W_{F}\right) & \left(M W_{4}-M W_{F}\right)
\end{array}\right]\left[\begin{array}{c}
\dot{n}^{S 1} \\
\dot{n}^{S 2} \\
\dot{n}^{S 3} \\
\dot{n}^{S 4}
\end{array}\right]=\left[\begin{array}{c}
\dot{n}_{\mathrm{Benz}}^{F} \\
\dot{n}_{\mathrm{CH}_{2}}^{F}+\dot{n}_{\left(\mathrm{CH}_{2}\right)_{n}}^{F} \\
\dot{n}_{\mathrm{CH}_{3}}^{F} \\
0
\end{array}\right]\right.
$$

where $\dot{n}^{S 1}$ is the vapor molar flow rate of surrogate component S1 (n-heptane). The first row of Eq. (2) is a 1:1 matching of the molar flow rate of the benzyl-type functional group of the full delumped fuel to the aromatic surrogate. The second row of Eq. (2) matches the molar flow rate sum of the $\mathrm{CH}_{2}$ and $\left(\mathrm{CH}_{2}\right)_{n}$ functional groups from the real fuel to that of the surrogate. The third row of Eq. (2) similarly matches the $\mathrm{CH}_{3}$ functional groups. The importance of using two n-alkanes, one light and one heavy, can be seen in the matching of the MW in the fourth row of Eq. (2). It should be noted that the two n-alkane surrogate species should be selected such that the fourth row of Eq. (2) can be satisfied during the majority of the vaporization process. If the S1 surrogate 
species is too heavy to satisfy the MW matching equation during early vaporization times where the fuel going into the gas phase is very light, a non-physical solution to the system of equations will occur, causing negative values of $\dot{n}^{S j}$ for the surrogate. A similar non-physical solution will result if the $S 2$ surrogate species is too light to satisfy the equation during late vaporization times. In cases where the MW constraint cannot be satisfied, the solution can be modified by adjusting the solution according to the conditions

$$
\left\{\begin{array}{l}
\text { if } \dot{n}^{S 1}<0, n^{S 1}=0 \\
\text { if } \dot{n}^{S 2}<0, n^{S 2}=0
\end{array}\right.
$$

with the remaining $\dot{n}^{j}$ recalculated from rows 1,2 , and 3 of Eq. (2).

\subsection{Calculation of combustion property targets}

The hybrid method is validated by comparing the calculated CPTs of the chemical surrogate to those calculated for the real fuel from the $\mathrm{DCM}$. The $\mathrm{MW}$ and $\underline{\mathrm{H} / \mathrm{C} \text { ratio }}$ are calculated using linear blending rules based on the mole fractions of each species. DCN is calculated using the functional group regression developed in [19]. Because insufficient data currently exists for the TSI of pure cycloalkane components, it is not possible to calculate the TSI of the full delumped fuel. Therefore, accuracy of the TSI for the chemical surrogate is evaluated using comparison to typical ranges of TSI in kerosene fuels. The TSI of the surrogate is calculated using linear blending rules for pure components [37], [38], [39], [40] .

\section{Results and discussion}

For the simulations in this section, the CA-DQMoM with node approximation and delumping model for droplet vaporization was paired with the new surrogate FGM method in order to calculate the time dependent CPTs as the droplet vaporized. The results of the hybrid model demonstrate its effectiveness as a method for evaluating the effects of preferential vaporization on combustion behavior, a current unsolved problem in combustion simulations [23], [24]. Additionally, because the hybrid method uses a continuous thermodynamics droplet vaporization model instead of a physical surrogate, the computational efficiency of the physical portion of the model is not limited by the number of components required to accurately model physical properties of the fuel. For the results presented here, the combined CA-DQMoM and FGM methods will be referred to as the "hybrid" method. This hybrid method was validated against the "exact" method which models droplet vaporization using the full DCM and computes the gas-phase CPTs from the full discrete fuel.

\subsection{Kerosene droplet}

The test conditions for the vaporization models were a $50 \mu \mathrm{m}$ droplet of kerosene ( $n=36$ species) at $300 \mathrm{~K}$ exposed to gas at $500 \mathrm{~K}$ and 5 bar [30]. The far-field gas composition was assumed to be $100 \%$ air throughout the simulation. The initial mole fractions and normal boiling temperatures were obtained from [2] for the 36 discrete species in kerosene comprised of $n$-alkanes, iso-alkanes, benzenes, and cycloalkanes. For all CADQMoM node approximation results shown below, $N=3$ nodes were used. For all figures in this section, the final time shown corresponds to the time at which $99.7 \%$ of the droplet has vaporized, by volume.

The accuracy of the CA-DQMoM vaporization model has been previously verified [25]. Figures 2 and $\underline{3}$ shows the evolution of two physical fuel properties with time, the mean boiling temperature and liquid density of the droplet, calculated using CA-DQMoM with delumping and the full DCM. The accuracy with which CA-DQMoM with delumping calculates the physical properties of the fuel throughout the vaporization process demonstrates the advantages of using this computationally efficient moment method for the physical portion of the hybrid model. Surrogates which are designed to match only chemical combustion properties are often unable to 
emulate the density of the real fuel because cycloalkanes are omitted from the surrogate composition [19], further emphasizing the advantage of using hybrid models to accurately model physical properties.

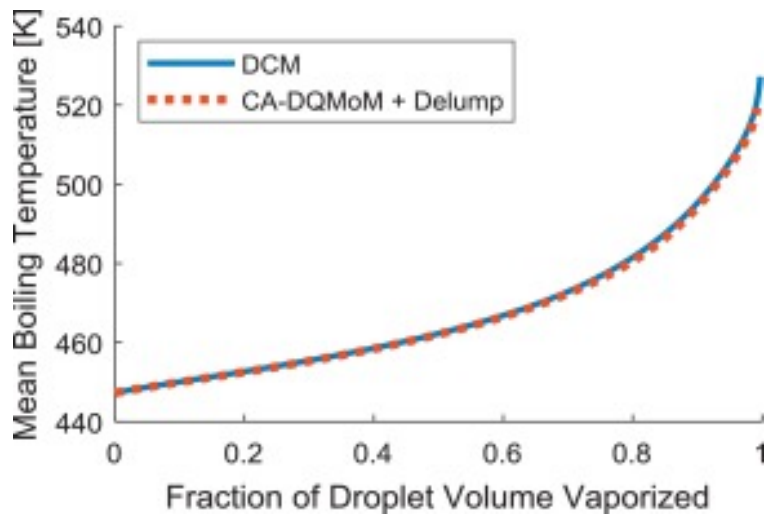

Fig. 2. Evolution of mean boiling temperature of the liquid droplet during the vaporization process, calculated using CADQMoM with node approximation and delumping, compared to the full DCM.

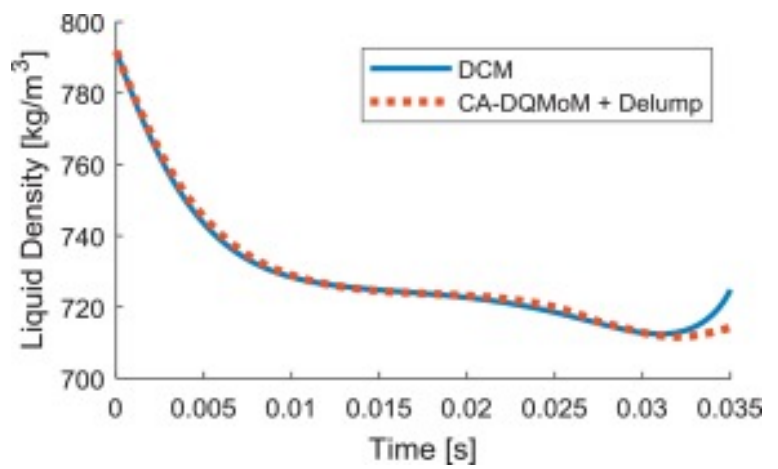

Fig. 3. Evolution of density of the liquid droplet during the vaporization process, calculated using CA-DQMoM with node approximation and delumping, compared to the full DCM.

The hybrid method utilizes FGM on the output of the CA-DQMoM with delumping model to successfully formulate a time-dependent chemical surrogate composition that matches the functional group distribution of the full fuel throughout the droplet vaporization process. Figure 4 shows the surrogate composition evolution calculated using the hybrid method. At the final time, the mole fraction of the light alkane ( $n$-heptane) has gone to zero invoking the conditions of Eq. (3) since MW can no longer be matched exactly. Figure 5 shows the instantaneous mole fraction evolution for each functional group with time. The agreement of functional group distribution is excellent between the chemical surrogate (hybrid) and the full fuel (exact) throughout the droplet vaporization process. These functional groups were determined to have the most significant impact on CPTs [19] and matching chemical functional groups is highly correlated to matching combustion behavior [7]. 


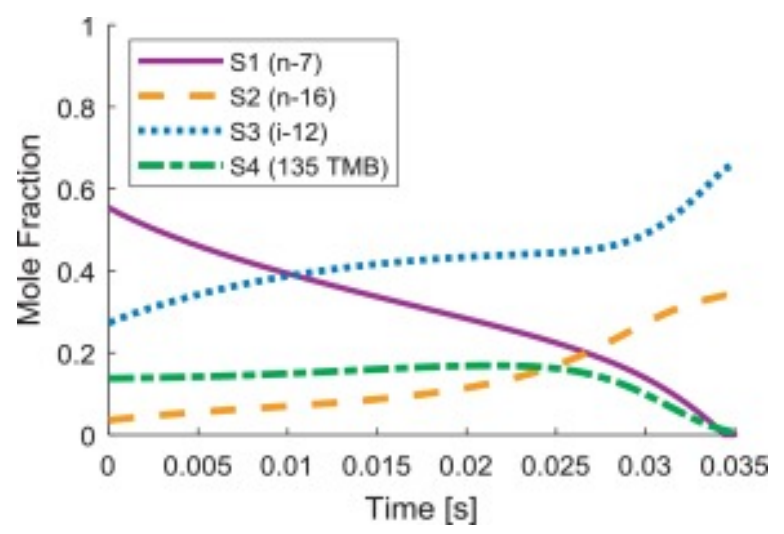

Fig. 4. Composition of the surrogate entering the gas phase vs. time.

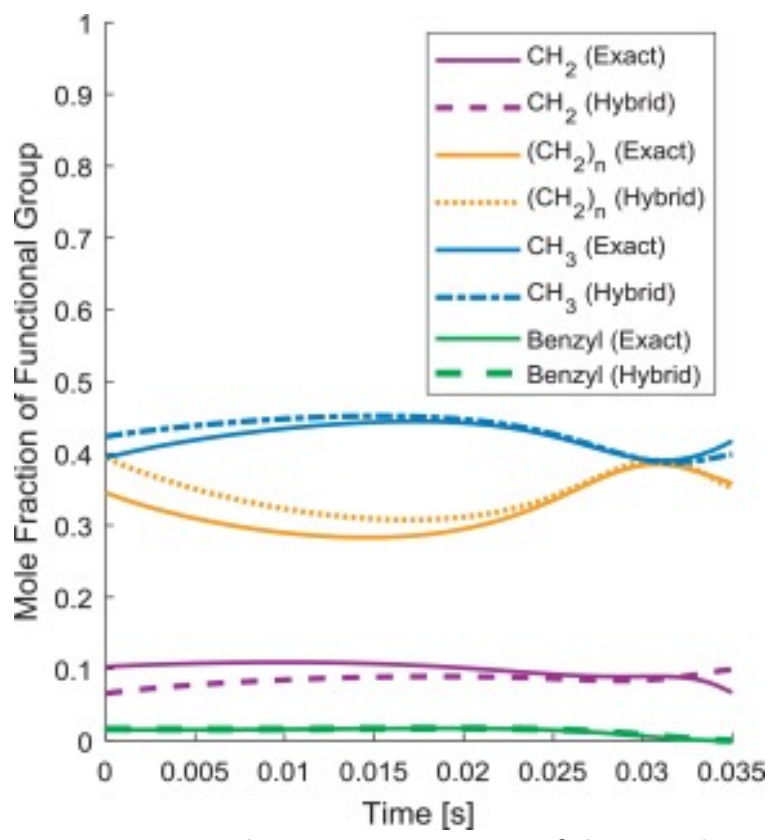

Fig. 5. Functional group composition of the gas phase source term over time calculated using the hybrid and exact methods.

The hybrid method also demonstrated excellent agreement with the exact method for calculation of transient, vaporization-dependent combustion properties. Figure 6 depicts the instantaneous CPTs of the fuel as it enters the gas phase calculated using the hybrid and exact methods. The horizontal lines in Fig. 6 show the properties of the pre-vaporized kerosene fuel for reference (it should be noted that the exact and hybrid graph lines do not approach the pre-vaporized line since the CPTs are calculated on an instantaneous basis for the fuel entering the gas phase). As expected, matching of the functional group distribution (ig. 5 ) leads to accurate matching of CPTs (Fig. 6), despite the added complexity of vaporization-dependence. The results presented here are consistent with previous studies which have observed large variations in CPTs due to preferential vaporization and distillation effects [19], [23]. 

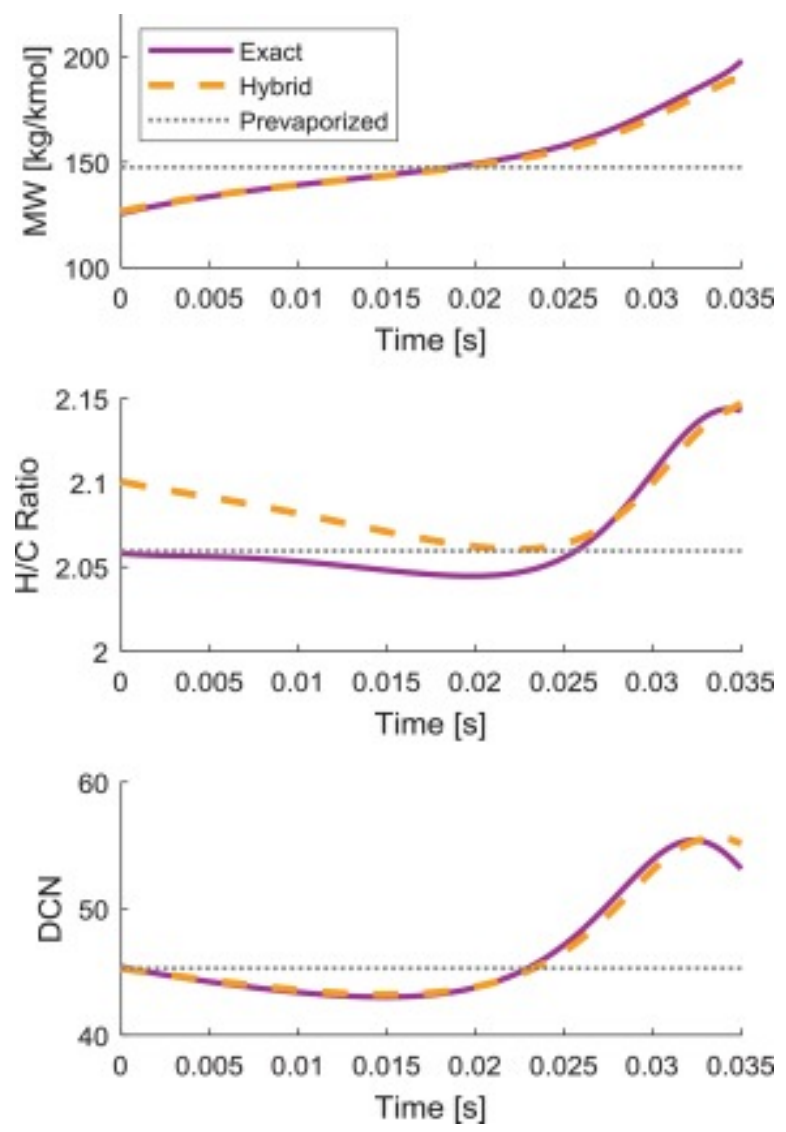

Fig. 6. Vaporization dependent CPTs of the instantaneous gas phase source term over time calculated using the hybrid and exact methods.

The TSI for the instantaneous chemical surrogate composition, calculated using the hybrid method, is shown in Fig. 7. As previously mentioned, TSI cannot be fully verified by the exact method due to lack of pure component data for cycloalkanes. However, there is promise in the ability of the hybrid method to predict sooting characteristics since the TSI values for the kerosene chemical surrogate in Fig. 7 demonstrate consistency with typical TSI ranges of 15-26 for kerosene fuels [11]. The TSI drops below the typical ranges for kerosene at the later times, by which most aromatics have vaporized (see Fig. 4), demonstrating a potential effect of vaporization on sooting tendency.

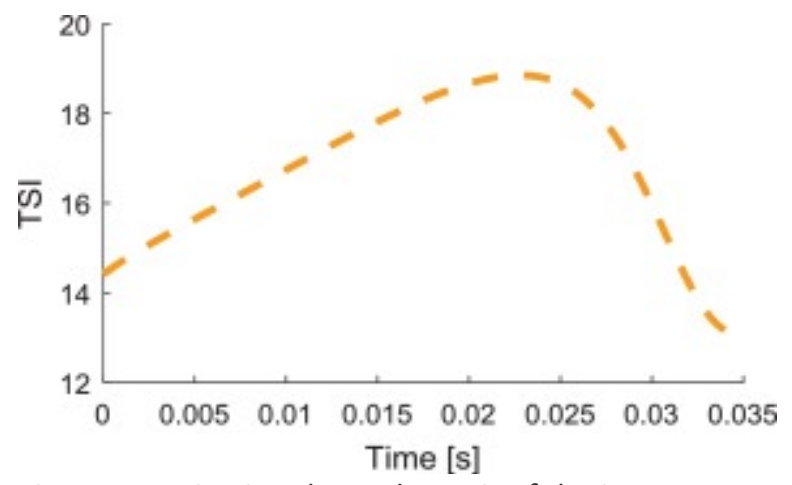

Fig. 7. Vaporization dependent TSI of the instantaneous gas phase source term over time calculated using the hybrid method.

The overall accuracy of the hybrid method in emulating the CPTs of the full fuel is excellent. As shown in Fig. 8 , the relative error between the hybrid and exact methods remained below $5 \%$ for all CPTs. The minimal error 
demonstrates the robustness of the hybrid method to represent time-dependent combustion behavior due to vaporization effects.

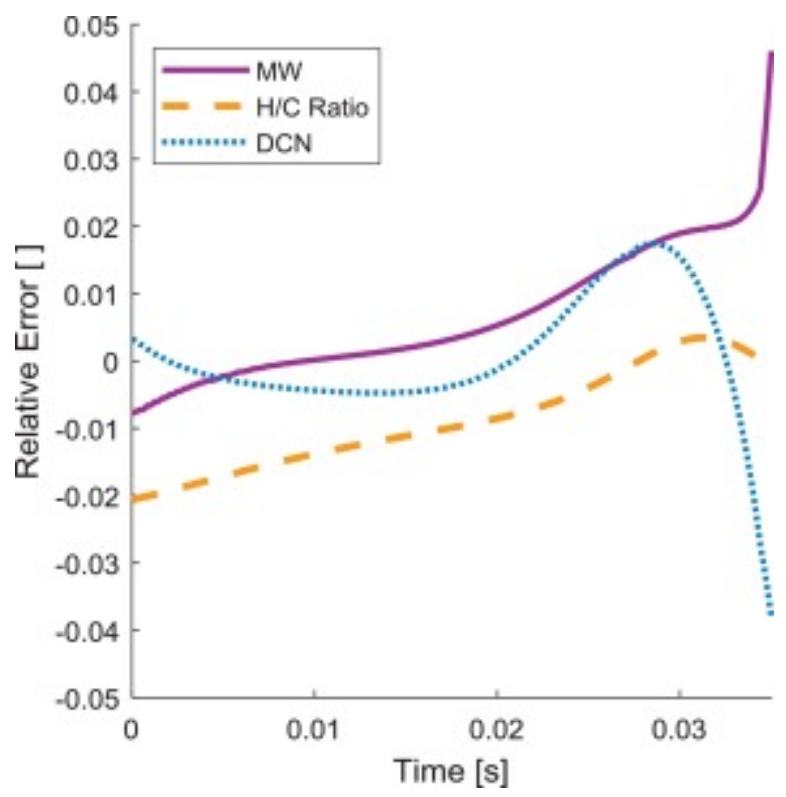

Fig. 8. Relative error for the vaporization dependent CPTs calculated using the hybrid method compared to the exact method.

As previously demonstrated [25], CA-DQMoM is a very computationally efficient method for solving multicomponent droplet vaporization relative to DCMs. Calculation of the chemical surrogate composition using FGM requires negligible computation time since the method only requires the solution of four algebraic equations, making it very computationally attractive. The hybrid approach using CA-DQMoM with node approximation and delumping combined with FGM achieves a computational savings of $77 \%$ compared to a full DCM for a droplet of 36 species. For a droplet with more than 36 species, even greater computational savings would be expected [25].

\section{Conclusions}

A computationally efficient, droplet vaporization-chemical surrogate hybrid approach has been developed for application in combustion CFD simulations to account for vaporization-dependent combustion behavior. The physical portion of the hybrid approach uses the Coupled Algebraic-Direct Quadrature Method of Moments (CADQMoM) with node approximation to solve for the evolution of a vaporizing multicomponent liquid fuel droplet. A delumping method is applied to reconstruct the information on all discrete fuel species, including the species molar flow rates into the gas phase. The output of the physical vaporization model is linked to the chemical surrogate through a novel functional group matching (FGM) method. The approach calculates the instantaneous molar flow rate of each surrogate species by matching the molecular weight and the distribution of key combustion functional groups $\left(\mathrm{CH}_{2},\left(\mathrm{CH}_{2}\right)_{n}, \mathrm{CH}_{3}\right.$ and benzyl-type) within the surrogate to that of the full fuel.

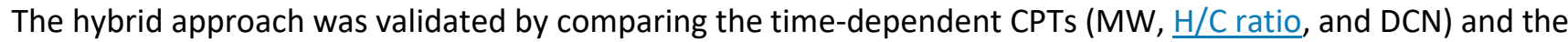
functional group distribution of the surrogate to those calculated using an exact method. The exact method employed a DCM for droplet vaporization and calculated CPTs based on all discrete fuel species. The hybrid approach showed excellent agreement with the exact method and the relative error for all CPTs was below $5 \%$ throughout the droplet vaporization process. The use of CA-DQMoM with delumping for the physical portion of the hybrid approach resulted in a 77\% savings in computation time compared to the DCM of the exact method. 
The computation time for FGM was negligible, making it an attractive method for vaporization-dependent surrogate formulation. The hybrid approach presented in this paper is an important step towards understanding the effects of preferential vaporization on combustion behavior. Future work will focus on applying the hybrid method to other types of fuel and extending the vaporization model to conditions with non-ideal vapor-liquid equilibrium using the Peng-Robinson equation of state and new property correlations for those conditions.

\section{Acknowledgments}

Funding from Marquette University's College of Engineering Legacy Initiative Seed Grant Program is gratefully acknowledged.

\section{References}

[1] N. Doué Modélisation de l'évaporation de gouttes multi-composants Ph.D. Thesis L'École Nationale Supérieure (2005)

[2]C. Laurent Développement et validation de modèles d'évaporation multi-composant Ph.D. Thesis Universite de Toulouse, I'Institut Supérieur de l'Aéronautique et de l'Espace (2008)

[3] H.T. Mayfield Armstrong Laboratory Report: JP-8 Composition and Variability (1996), pp. 1-26

[4] T.M. Lovestead, J.L. Burger, N. Schneider, T.J. Bruno Energy Fuels (2016), pp. 10029-10044

[5] S.M. Sarathy, G. Kukkadapu, M. Mehl, et al. Proc. Combust. Inst., 35 (2015), pp. 249-257

[6] P.B. Govindaraju, M. Ihme Int. J. Heat Mass Transf., 102 (2016), pp. 833-845

[7] F.L. Dryer Proc. Combust. Inst., 35 (2015), pp. 117-144

[8] S. Dooley, Won S.H., M. Chaos, et al. Combust. Flame, 157 (2010), pp. 2333-2339

[9] Ra Y., R.D. Reitz Combust. Flame, 158 (2011), pp. 69-90

[10] K. Anand, Ra Y., R.D. Reitz, B. Bunting Energy Fuels, 25 (2011), pp. 1474-1484

[11] S. Dooley, Won S.H., J. Heyne, et al. Combust. Flame, 159 (2012), pp. 1444-1466

[12] C.J. Mueller, W.J. Cannella, T.J. Bruno, et al. Energy Fuels, 26 (2012), pp. 3284-3303

[13] A. Krishnasamy, R.D. Reitz, W. Willems, E. Kurtz Proceedings of the SAE 2013 World Congress \& Exhibition (2013) 2013-01-1092

[14] Chang Y., Jia M., Li Y., et al. Combust. Flame, 162 (2014), pp. 3785-3802

[15] Kim D., J. Martz, A. Violi Combust. Flame, 161 (2014), pp. 1489-1498

[16] Ra Y., R.D. Reitz Combust. Flame, 162 (2015), pp. 3456-3481

[17] Chen X., E. Khani, Chen C.P. Fuel, 182 (2016), pp. 284-291

[18] Kim D., J. Martz, A. Abdul-Nour, Yu X., M. Jansons, A. Violi Combust. Flame, 179 (2017), pp. 86-94

[19]Won S.H., F.M. Haas, S. Dooley, T. Edwards, F.L. Dryer Combust. Flame, 183 (2017), pp. 39-49

[20] S. Dooley, Won S.H., S. Jahangirian, et al. Combust. Flame, 159 (2012), pp. 3014-3020

[21] N. Doué, P. Le Clercq, M. Aigner International Conference on Liquid Atomization and Spray Systems (2006)

[22] P. Le Clercq, N. Dou 47th AIAA Aerospace Science Meeting (2009)

[23] J.A. Lefkowitz, F.M. Haas 10th U. S. National Combustion Meeting (2017)

[24] A. Stagni, L. Esclapez, P. Govindaraju, A. Cuoci, T. Faravelli, M. Ihme Proc. Combust. Inst, 36 (2017), pp. 2483-2491

[25] A.Y. Cooney, S.L. Singer Fuel, 212 (2018), pp. 554-565

[26]S.L. Singer Int. J. Heat Mass Transf, 103 (2016), pp. 940-954

[27] L.A. Dombrovsky, S.S. Sazhin Int. Commun. Heat Mass Transf, 30 (2003), pp. 787-796

[28] G.J. Brereton Int. J. Heat Mass Transf, 60 (2013), pp. 512-522

[29]A. Bruyat, C. Laurent, O. Rouzaud 7th International Conference on Multiphase Flow (2010)

[30]C. Laurent, G. Lavergne, P. Villedieu Int. J. Multiph. Flow, 36 (2010), pp. 51-59

[31]C. Laurent, G. Lavergne, P. Villedieu Comptes Rendus Mécanique, 337 (2009), pp. 449-457 
[32] J. Tamim, W.L.H. Hallett Chem. Eng. Sci, 50 (1995), pp. 2933-2942

[33] W.L.H. Hallett Combust. Flame, 121 (2000), pp. 334-344

[34] Ra Y., R.D. Reitz Int. J. Multiph. Flow, 35 (2009), pp. 101-117

[35] P.L.C. Lage Comput. Chem. Eng., 31 (2007), pp. 782-799

[36] D.L. Marchisio, R.O. Fox J. Aerosol Sci., 36 (2005), pp. 43-73

[37] D.B. Olson, J.C. Pickens, R.J. Gill Combust. Flame, 62 (1985), pp. 43-60

[38] R.J. Gill, D.B. Olson Combust. Sci. Technol., 40 (1984), pp. 307-315

[39] A. Mensch, R.J. Santoro, T.A. Litzinger, Lee S.Y. Combust. Flame, 157 (2010), pp. 1097-1105

[40] Won S.H., F.M. Haas, A. Tekawade, et al. Combust. Flame, 165 (2016), pp. 137-143 